\title{
e-Phaïstos
}

e-Phaïstos

Revue d'histoire des techniques / Journal of the history

of technology

IX-2 | 2021

Quel objet pour quel musée?

\section{Obsolescence programmée?}

Les limites de la conservation d'un objet dans le musée

The Limits of the Conservation of an Object in the Museum or: Planned

Obsolescence?

\section{Bénédicte Rolland-Villemot}

\section{(2) OpenEdition}

\section{Journals}

Édition électronique

URL : https://journals.openedition.org/ephaistos/9669

DOI : 10.4000/ephaistos.9669

ISSN : 2552-0741

Éditeur

IHMC - Institut d'histoire moderne et contemporaine (UMR 8066)

Référence électronique

Bénédicte Rolland-Villemot, « Obsolescence programmée ? », e-Phaïstos [En ligne], IX-2 | 2021, mis en ligne le 26 octobre 2020, consulté le 28 octobre 2021. URL : http://journals.openedition.org/ephaistos/ 9669 ; DOl : https://doi.org/10.4000/ephaistos.9669

Ce document a été généré automatiquement le 28 octobre 2021.

Tous droits réservés 


\section{Obsolescence programmée?}

Les limites de la conservation d'un objet dans le musée

The Limits of the Conservation of an Object in the Museum or: Planned

Obsolescence?

Bénédicte Rolland-Villemot

1 «Commençons par une évidence : le sort de tout objet fait de la main de l'homme est de subir des modifications, de se dégrader et finalement de disparaître » (Rolland-Villemot 2015b). La forme initiale de l'objet correspond à des besoins, des fonctions et des goûts datés et situés. La modification de ceux-ci au fil du temps peut entraîner soit l'adaptation de l'objet, soit conduire à son abandon ou à sa destruction. Cela vaut pour les fonctions symboliques, comme pour les fonctions pratiques.

\section{Les musées de société, d'anthropologie d'industrie mais aussi d'art contemporain sont confrontés à l'obsolescence programmée}

2 La problématique de l'obsolescence technologique ou programmée ou, autrement dit, le renouvellement incessant des produits mis en circulation sur le marché, affecte en effet une part importante de la production des objets contemporains susceptibles d'être collectés par les musées. La question de savoir où commence et où finit un objet n'est pas absurde voire futile, surtout quand on considère qu'il est élément d'un système dans lequel il remplit une fonction technique et une fonction symbolique. La multiplicité des sources que recouvrent les activités techniques justifie l'intérêt porté aux recherches sur les archives matérielles de l'histoire des techniques que sont les collections des musées. Le musée ne conserve, à proprement parler, que de la matière ; comment transmettrait-il des savoir-faire qui ne se transmettent que dans la pratique, dans le cadre du groupe de travail, à la différence des savoirs enseignés formellement, en milieu scolaire par exemple? Le musée n'a pas, à notre sens, comme objectif principal de diffuser des connaissances ou techniques, d'acculturer à la technique, ou de permettre une meilleure adaptation des gens à leur environnement technique. En 
effet, l'objet d'art est le produit d'une approche esthétique, mais également technique par les processus qui sont mis en cause lors de sa création ou de sa production.

Les objets de notre quotidien sont de plus en plus des objets manufacturés, fabriqués en série et leur durée est donc limitée dans le temps, soit pour des raisons commerciales, soit du fait de la dégradation des matériaux. L'obsolescence fonctionnelle correspond au fait qu'un produit ne répond plus aux nouveaux usages attendus, pour des raisons techniques (par exemple l'incompatibilité avec de nouveaux équipements), réglementaires et/ou économiques. L'obsolescence d'évolution renvoie au fait qu'un produit s'éloigne des envies des utilisateurs qui souhaitent acquérir un nouveau modèle du fait d'une évolution de fonctionnalité ou de design. L'obsolescence programmée (parfois aussi appelée "désuétude planifiée ») est le nom donné par abus de langage à l'ensemble des techniques visant à réduire la durée de vie ou d'utilisation d'un produit afin d'en augmenter le taux de remplacement.

\section{La désuétude est la caractéristique essentielle de l'activité muséale et de la patrimonialisation}

4 En effet, les objets entrent dans les musées parce qu'ils n'ont plus d'utilité dans leur culture d'origine. C'est l'obsolescence culturelle ou technique qui fonde la valeur des collections muséales. Il faut donc en tenir compte. Cette désuétude dite "planifiée » a toujours existé : les phénomènes de mode influencent la fabrication et l'usage des objets dans nos sociétés. Ils se sont accélérés avec l'apparition de la société dite de consommation et les progrès de l'industrialisation comme de la chimie qui ont permis de produire à moindre coût des objets plus nombreux et dégradables pour satisfaire les besoins fluctuants de la clientèle. La réussite technique et le succès du fameux bas en nylon sont de bons exemples de la position de notre société contemporaine face à la durabilité des biens de consommation. Mis sur le marché par Dupont de Nemours dans les années 1940, le bas nylon était si résistant que les ventes s'effondrèrent, faute de besoin de renouvellement. En modifiant la formule (notamment en réduisant le dosage de certains additifs destinés à protéger le polymère des rayons UV), le bas nylon est devenu plus fragile. Il file et, contrairement à son prédécesseur en soie ou en laine, n'est pas reprisable; il faut nécessairement le remplacer (Rolland-Villemot $2013: 29$; Delahaye 2014).

5 Ce nouveau mode de production et de consommation provoque des changements culturels dans le rapport aux objets. La tension entre l'éphémère et la conservation fonde le patrimoine et ses métiers. Qu'est-ce qui a été choisi dans cet objet pour être conservé ? Quelle part de son apparence au moment où il a été confié au musée est importante ? Faut-il maintenir l'objet dans sa matérialité initiale ou bien l'apparence de l'œuvre ou de l'objet? Est-ce l'intentionnalité ou la matérialité des collections qui importe? Cette problématique oblige à repenser de manière radicale le cadre déontologique de la conservation-restauration et donc de l'inaliénabilité des collections: Conserver à l'heure du consommable? Remplacement à l'identique, dématérialisation des collections? Quelle pérennité?

6 L'obsolescence d'un matériel conduit à une aporie : l'objet semble être condamné à ne plus pouvoir être présenté, ni à être intelligible. L'objet dépendant totalement d'une technique et d'un moment du développement industriel est voué à l'oubli et à la 
disparition: il est difficilement recyclable ou réutilisable.» Il n'existe donc pas de solution générique pour la conservation matérielle de ces objets quand ils deviennent des collections. L'évolution technique qui frappe d'obsolescence certains éléments de l'objet lui impose une typologie, une chronologie qui sont celles de la technique et non de l'objet et qui se trouvent épistémologiquement en contradiction avec la pérennité de la matière que contient la notion de conservation ».

\section{Il faut donc réaffirmer la part technique et culturelle de tout objet}

7 «L'activité technique, en édifiant le monde des objets techniques et en généralisant la médiation objective entre homme et nature, rattache l'homme à la nature selon un lien beaucoup plus riche et mieux défini que celui de la réaction spécifique de travail collectif. Une convertibilité de l'humain en naturel et du naturel en humain s'institue à travers le schématisme technique" (Simondon 1958 :245). Gilbert Simondon, dans Du mode d'existence des objets techniques, souligne dès 1958 la nécessité de s'intéresser aux modes d'existence dans l'espace et dans le temps des objets et des œuvres d'art. L'obsolescence est sans doute un problème à résoudre, un ensemble de conditions socio-économiques et techniques nouvelles qui affecte l'objet de manière extérieure.

8 L'obsolescence est à envisager comme un mode d'existence de la probable difficulté à établir le lien entre la pensée et le geste qui a donné naissance à l'objet et à des systèmes socio-techniques dont dépend l'existence matérielle de celui-ci. L'objet découle alors des contenus psychosociaux du moment et du système de pensée technique auquel il appartient. Simondon, dans Imagination et invention, définit deux types d'œuvres d'art en fonction de ces systèmes de pensée: l'œuvre analytique et l'œuvre synthétique qui sont liées. Elles dépendent dialectiquement du système technologique dans lequel elles ont été produites. Ainsi, le téléviseur cathodique irrémédiablement remplacé par l'écran plat est un objet synthétique dont la désuétude est planifiée ; les pièces de rechange, par exemple les lampes cathodiques, ne sont plus fabriquées, faute d'utilité économique. Pour Simondon, même les techniques les plus ordinaires sont traversées par des volontés et des intentions humaines (Simondon 2006).

9 L'introduction de certains objets techniques rend désuètes certaines valeurs ou formes matérielles de vie. De plus, certaines performances techniques rencontrent l'hostilité sociale et sont rejetées ou corrigées. L'obsolescence peut alors faire sens. Elle est le destin même de ces objets. Ces objets existent donc sur un mode problématique. Ce questionnement contemporain doit faire partie de la réflexion d'un musée de société qui veut rendre compte de la réalité contemporaine.

10 Cette obsolescence des collections réunies et conservées forme une remise en cause de la notion d'intentionnalité, de pérennité et d'authenticité. Elle appelle à une réinstauration de l'objet dans son contexte technique. La matière compte autant que la forme. La matérialité éphémère de ces objets signifie leur valeur culturelle. Ce caractère éphémère ne s'oppose pas nécessairement à la pérennité qui n'est jamais absolue. L'obsolescence est relative à un système sociotechnique qui, en se transformant, peut réactualiser des objets périssables. 
11 La diversification sans limite des matériaux, des techniques et des procédés, inhérente à l'art contemporain, introduit une nouvelle problématique : celle de l'obsolescence technologique des matériels. Alors qu'en pratique, les professionnels sont constamment confrontés à cette question, l'étude des phénomènes d'obsolescence technologique dans le contexte de l'art contemporain et de la conservationrestauration constitue un champ disciplinaire encore émergent. Avec l'avènement du numérique, l'obsolescence technologique fait peser sur les œuvres une lourde menace : des technologies révolues font que certaines œuvres ne peuvent plus être « réactivées». Issus des beaux-arts traditionnels, les cadres déontologiques de la conservation et de la restauration sont parfois décalés face à la gestion des phénomènes d'obsolescence technologique.

12 Nous sommes dans l'ère des industries culturelles. Pour Walter Benjamin, l'œuvre d'art est à l'époque de sa reproductibilité technique: le développement technique a profondément changé notre rapport aux productions culturelles. L'évolution des moyens de production artistique, c'est-à-dire le passage du stade de la production artisanale à celui de la grande technique, transforme l'auteur en producteur parmi d'autres (Schimder 2014):

«À la plus parfaite reproduction, il manquera toujours une chose : le hic et nunc de l'œuvre d'art, l'unicité de son existence au lieu où elle se trouve». "Tout ce qui relève de l'authenticité échappe à la reproduction » (Benjamin 1939).

\section{L'œuvre d'art reproduite techniquement et reproductible a perdu son aura}

13 Par sa présence, l'œuvre est d'abord unique et possède une valeur cultuelle. Avec la reproduction, on va passer d'une valeur cultuelle à une valeur d'exposition. Notre rapport aux œuvres change donc. Hegel, dans l'Esthétique, avait déjà annoncé cette transformation en nous disant que « l'œuvre d'art, dans sa destination suprême est une chose du passé ", formule que l'on résume souvent, au risque de faire un contresens par «l'art est mort». Hegel ne nous dit pas simplement que l'art est mort, il nous dit que l'art est pour nous quelque chose du passé, " du moins dans sa destination suprême » (Hegel (1818-1829) 1997 :549).

14 Ceci signifie l'achèvement d'une époque particulière, celle d'une authentique sacralité des œuvres. Nous ne vivons plus au temps où les œuvres d'art sollicitaient la vénération, parce qu'elles incarnaient d'une manière ou d'une autre la présence du divin. Nous ne nous agenouillons plus nécessairement avant de pénétrer dans un temple. Nous sommes éloignés des intentions et des sentiments des hommes de Lascaux. Ce qui était Dieu dans un temple devient une statue dans un musée. Un portrait ou une icône dans une église deviennent un tableau dans une galerie... Notre rapport à l'art a changé.

15 À l'ère du numérique, une stratégie globale est d'autant plus urgente qu'il est impératif de rattraper les effets du non-renouvellement générationnel des pratiques culturelles et d'agir sur leurs transmissions. 
16 Peut-être, en s'intéressant à l'histoire matérielle et en analysant les collections de musées comme des archives matérielles de l'histoire des techniques et en essayant de comprendre comment, en France, des tentatives de construction d'unicité autour des œuvres pour réconcilier la matière et l'image ont existé, on pourra retrouver ce « qui a été ». Les collections des musées ne sont que des fragments, des restes, des ruines, des archives matérielles de sociétés ou de civilisations proches ou lointaines mais appartenant déjà au passé. Il faut revenir au "Quoi de l'œuvre » comme le suggère Heidegger (Heidegger 1987, Balazut 2010).

\section{Depuis un demi-siècle, l'univers technique a été métamorphosé}

17 Jusqu'en 1960, il reposait sur la métallurgie, la mécanique, les réseaux de distribution. Aujourd'hui, les objets et les systèmes techniques sont devenus numériques. Vient le temps de l'open innovation. Mais la physique nucléaire, les algorithmes et Von Neumann furent également à l'origine du projet Manhattan et de la bombe d'Hiroshima. L'innovation, la technique possèdent une troublante dualité.

Dans le numéro Hors-série du Monde (6 juillet 2015) consacré à l'Innovation, Yves Michaud écrit jusqu'où irons-nous : "Notre époque est donc dans la puissance et la rapidité ». Cela pose des problèmes : l'adaptation à des changements très rapides et l'éventualité de la modification de la nature humaine elle-même. Mais contrairement à Jankélévitch, pour Francis Bacon, le temps abîme les choses, de sorte que l'innovation doit exister pour empêcher la lente destruction ${ }^{1}$. «Si le temps change les choses pour le pire et que la sagesse et le conseil ne les modifient pas pour le meilleur, quelle sera alors la fin? » (Schrepel Aghion 2018).

19 Selon Étienne Klein (Le Monde, hors-série déjà cité), au temps des cathédrales, il s'agissait d'associer des multiples savoirs artisanaux et non d'appliquer un corpus théorique préalable. Par la suite, les inventions ont dépassé ce stade et résultent directement de découvertes scientifiques et, selon lui, c'est ainsi que la technologie a vraiment pris le pas sur la technique. Günther Anders, dans l'Obsolescence de l'homme, écrit que l'homme se sent écrasé par les pouvoirs de la science que l'innovation donne à la technique. Pour lui, nous sommes dans une angoisse de l'homme prométhéen : «La honte qui s'empare de l'homme devant l'humiliante qualité des choses qu'il a lui-même fabriquées. Il a honte d'être devenu plutôt que d'avoir été fabriqué» (Anders 1956). Cette honte est accentuée aujourd'hui car notre rapport aux objets a changé. Nous ignorons presque tout des objets de notre quotidien; ils sont devenus illisibles: Comment sont-ils fabriqués? Comment fonctionnent-t-ils? À quoi servent-ils vraiment?

20 À cela s'ajoute un rapport quasi-magique à la technologie. Nous ne savons plus réparer nos objets technologiques. Si mon téléphone portable ne marche plus, je le jette; si ma voiture tombe en panne, j'appelle un numéro vert. Que je sois ingénieur (régime de la technique) ou conservateur du patrimoine, mon rapport à l'objet est le même. Nous avons technologisé le monde.

21 Cette technologisation de la culture est condamnée par Heidegger, qui rejette bien évidemment «l'objectivation technico-scientifique de la nature ». La nature se joue de l'homme mais ce n'est pas l'homme qui se joue de la nature. Heidegger entrevoit les 
excès d'un comportement aberrant de l'homme, d'un comportement " commettant " où l'homme provoque ostensiblement la nature (Heidegger 1987). Cette pensée développée de la nature se trouve chez Heidegger car, dans Être et Temps, l'ambiguïté reste présente : appréhende-t-il la nature à travers l'usage et la visée d'ustensilité de la technique? Mais depuis Hiroshima, le nucléaire pointe son épée de Damoclès (docteur Fol Amour von Neumann) et surtout depuis Fukushima, la honte prométhéenne a resurgi. C'est alors l'autonomie de la technique l'enjeu d'aujourd'hui.

\section{Mais tout ceci est-il irréversible ? Ne peut-on restaurer le régime de la pratique?}

L'historien Fernand Braudel propose, en réponse à une critique de Lévi-Strauss contre l'histoire, la longue durée comme structure. Il développe la notion de temporalité. Dans un même espace-temps, plusieurs temporalités existent. Pour lui comme pour Lucien Febvre, l'historien n'étudie pas un objet mais un problème. Nous proposons donc, à travers les objets techniques que sont les collections de musées, d'étudier le problème du rapport à la technique et des différentes temporalités techniques que recouvrent les études autour des collections patrimoniales et de leur rapport à la conservationrestauration : histoire matérielle de l'objet, imprescriptibilité, irréversibilité, pratique des techniques, culture numérique, la matière à l'œuvre dans les biens culturels : comment l'étudier, la détecter, la restaurer matériellement et intellectuellement pour la rendre compréhensible aujourd'hui pour demain. La transmission d'un monde disparu de la technique de façon irréversible est à ce prix, le retour à la matière (Rolland-Villemot 2015a).

23 Jankélévitch, dans L'irréversible et la nostalgie, analyse ces deux notions dans leur rapport à la mort, à l'ineffable, le presque rien, cette esthétique de l'éphémère qui lui est si chère (Jankelevitch 1974/2011).

Qu'est-ce que la nostalgie sinon une mélancolie humaine rendue possible par la conscience de quelque chose d'autre, d'un ailleurs, d'un contraste entre passé et présent? Et cette nostalgie n'est-elle pas aussi provoquée essentiellement par l'irréversibilité du temps? Car on ne saurait remonter le cours du temps, tel est l'obstacle insurmontable qu'il oppose à nos entreprises. C'est notre impuissance devant cette impossibilité qui fait toute l'amertume de la nostalgie et l'absurdité des chimères du rajeunissement. La nostalgie n'est pas le mal. On peut toujours revenir à son point de départ, à son lieu natal (l'espace se prête docilement à toutes nos allées et venues) mais il est impossible de redevenir celui qu'on était au moment du départ. Si le temps s'oppose irréductiblement à la rétrogradation, il ouvre devant nous une carrière infinie à la liberté. L'homme peut s'ouvrir à l'idée du futur et confirmer ce que le temps affirme. Se servant d'exemples tirés de la vie quotidienne aussi bien que d'œuvres poétiques, philosophiques ou musicales, Vladimir Jankélévitch démontre enfin de compte que l'irréversible n'admet qu'un seul remède: le consentement joyeux de l'homme à l'avenir, au futur (Jankélévitch 1974).

Donc l'irréversibilité de qui a été (le régime de la pratique²) est par ce qu'il a été présent et imprescriptible. Comme les collections de musées: c'est leur lecture technique qu'il faut construire pour réinvestir le monde de la technique et de la 
matérialité quand tout nous fait croire que le monde est dématérialisé ? Le cloud est-il si dématérialisé alors qu'il est énergivore?

L'objet technologique jetable ne fait-il pas rimer innovation avec pollution et accumulation de déchets? Le « rien ne se jette, tout se transforme » attribué à Lavoisier peut-il expliquer l'économie circulaire, l'objet durable, la soutenabilité culturelle que représente l'objet patrimonial dans sa durabilité matérielle, son authenticité technique qui s'inscrit dans la temporalité des techniques : régime de la pratique (au temps de sa production), régime de la technique (au temps de sa production et de sa conservation), régime de la technologie (le processus de conservation et la chaîne patrimoniale).

\section{Et l'obsolescence de l'objet numérique ? Peut-on envisager une conservation technologique?}

Elle consiste à préserver l'environnement technique du fonctionnement du système, y compris le système d'exploitation, les logiciels d'application originaux, les lecteurs de support, etc. C'est ce que l'on appelle parfois la solution du "musée informatique ». La conservation technologique se veut davantage une stratégie de récupération en cas de désastre.

Elle permet de faire face à l'obsolescence des supports, en supposant que ceux-ci ne se sont pas dégradés au point d'être illisibles. Elle permet d'agrandir la fenêtre d'accès pour des supports et des formats de fichier périmés mais elle mène ultimement à une impasse puisqu'aucune technologie obsolescente ne peut être maintenue indéfiniment en état de fonctionnement. Le maintien d'une technologie obsolescente en état d'utilisation exige un investissement considérable en équipement et en personnel.

Les musées de société conservent également des denrées pétrissables comme des pains ou des gâteaux rituels. Certains produits possèdent une date de péremption. Cela s'applique principalement aux aliments et aux boissons qui ont une date limite de consommation. Comment en assurer la conservation à long terme? La pérennité est alors une espérance!

\section{BIBLIOGRAPHIE}

ANDERS Gunther, L'obsolescence de l'homme, Traduit de l'allemand par Christophe David, Paris Éditions de l'encyclopédie des nuisances, (1956), 2002

BALAZUT Joël, « La thèse de Heidegger sur l'art », Nouvelle revue d'esthétique, n 5, 2010/1, p. $141-152$

BENJAMIN Walter, L'œuvre d'art à l'époque de sa reproductibilité technique, Paris, Gallimard, (1939), 2000

BREUIL Marie-Hélène et DAZORD Cécile (dir.), « Conserver l'art contemporain à l'ère de

l'obsolescence technologique », Technè, n 37, 2013 
DELAHAYE Lydie. « Le Musée, à La Recherche Du Contemporain », Vingtième Siècle. Revue d'histoire, n 123, 2014, p. 207-9 ; URL : http://www.jstor.org/stable/24673899

DURAND Anita, « Valeurs, compromis et polémiques », CeROArt [En ligne], 4 | 2009, mis en ligne le 14 octobre 2009 : http://ceroart.revues.org/1259

FREMEAUX Philippe, « Des produits conçus pour ne pas durer? », Alternatives économiques, $n^{\circ} 305$, septembre 2011

GARÇON Anne-Françoise, L'imaginaire et la pensée technique, Paris, Éditions Classiques Garnier, 2012

HEGEL Friedrich, L'esthétique ou philosophie de l'art, Paris, Livre de poche, 1818-1829, réed. 1997 HEIDEGGER Martin (trad. Wolfgang Brokmeier), «L'origine de l'œuvre d'art », in Chemins qui ne mènent nulle part, Gallimard, coll. « Tel », 1987

JANKELEVITCH Vladimir, L'irréversible et la nostalgie, Paris, Champ essais, (1974), 2011

ROLLAND-VILLEMOT Bénédicte, « Contemporain pour toujours ou comment conserver le consommable ", in Exposer, s'exposer : de quoi le musée est-il le contemporain ?, $2^{\mathrm{e}}$ rencontres scientifiques du MUCEM, 5-7 décembre 2013, p. 27-30 ; URL : https://fr.calameo.com/read/ 0023583766 b9352f 28667

ROLLAND-VILLEMOT Bénédicte, « Irréversibilité et nostalgie : Hiroshima mon amour, algorithmes, van Neumann, patrimoine culturel ou les trois régimes de la pensée opératoire », Techniques et musées, Hypothèses (en ligne), 2015 (a) ; URL : https://techmus.hypotheses.org/56 ROLLAND-VILLEMOT Bénédicte, «Les éphémères dans le catalogue des collections du Musées des civilisations de l'Europe et de la Méditerranée (MUCEM) », Fabula / Les colloques, Les éphémères, un patrimoine à construire, mis en ligne le 8 décembre 2015 (b) ; URL : https://www.fabula.org/ colloques/document2898.php

SCHIMDER Christine, « La dialectique de l'authenticité chez Walter Benjamin. Enjeux politiques et esthétiques ", Noesis, n²2-23, 2014, p. 29-42

SCHREPEL Thibault et AGHION Philippe, L'innovation prédatrice en droit de la concurrence, Paris, Éditions Bruylant, 2018

SIMONDON Gilbert, Du mode d'existence des objets techniques, Paris, Aubier-Montaigne, (1958), 2012 SIMONDON Gilbert, Imagination et invention, Chatou, Éditions de la transparence, 2006

\section{NOTES}

1. Francis Bacon, Essais de Morale et de Politique, publié en 1625.

2. Sur les régimes de technicité (ou régimes de la pensée opératoire): pratique, technique, technologie, voir Garçon 2012. 


\section{RÉSUMÉS}

Les musées de société, d'anthropologie, d'industrie mais aussi d'art contemporain sont confrontés à l'obsolescence programmée. La problématique de l'obsolescence technologique ou programmée ou, autrement dit, le renouvellement incessant des produits mis en circulation sur le marché, affecte en effet une part importante de la production des objets contemporains susceptibles d'être collectés par les musées. Cette problématique oblige à repenser de manière radicale le cadre déontologique de la conservation-restauration et donc de l'inaliénabilité des collections: conserver à l'heure du consommable? Remplacement à l'identique, dématérialisation des collections ? Quelle pérennité ? Faut-il maintenir l'objet dans sa matérialité initiale ou bien maintenir l'apparence de l'œuvre ou de l'objet? Est-ce l'intentionnalité ou la matérialité des collections qui importent?

Museums of society, museums of industrial anthropology but also museums of contemporary art are confronted with programmed obsolescence. The problem of technological or programmed obsolescence or, in other words, of the constant renewal of products put on the market, affects an important part of the production of contemporary objects likely to be collected by museums. This problem calls for a radical rethinking of the ethical framework of conservation-restoration and therefore of the inalienability of collections: conservation in an age of consumables? Identical replacement, dematerialization of collections? What is the durability? Should we maintain the object in its original materiality or maintain the appearance of the work or the object? Is it the intentionality or the materiality of the collections that matters?

\section{INDEX}

Mots-clés : histoire des techniques, musée, collection, muséologie, soutenabilité, technologie Keywords : history of technology, museum, collection, museology, sustainability, technology, society museum

\section{AUTEUR}

\section{BÉNÉDICTE ROLLAND-VILLEMOT}

Conservateur en chef du patrimoine, Bénédicte Rolland-Villemot est en charge des musées de société et des musées techniques au service des Musées de France au ministère de la Culture. Elle est enseignante à l'École du Louvre et à l'institut national du Patrimoine (INP). Ses travaux portent sur l'histoire matérielle des collections de musées, sur les rapports en l'histoire des techniques et les pratiques muséales et sur les collections muséales comme archives matérielles pour une histoire de techniques. 